TERRA. Revista de Desarrollo Local e-ISSN: 2386-9968

Número 8 (2021), 715-722

DOI 10.7203/terra.8.20357

IIDL - Instituto Interuniversitario de Desarrollo Local

\title{
Reseña. El desarrollo local en ámbitos rurales: experiencias y buenas prácticas para la empleabilidad y el emprendimiento
}

\author{
Pere Joan Quiles Lledó \\ Graduado en Ciencias Políticas y de la Administración Pública (Universidad de \\ Valencia, España) \\ pequille@alumni.uv.es

\section{Pablo Miguel Argudo} \\ Graduado en Ciencias Políticas y de la Administración Pública (Universidad de \\ Valencia, España) \\ pamiar@alumni.uv.es

\section{Guillem Gil Puig} \\ Graduado en Ciencias Políticas y de la Administración Pública (Universidad de \\ Valencia, España) \\ guigil2@alumni.uv.es
}

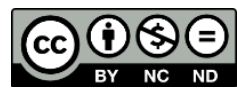

Esta obra se distribuye con la licencia Creative Commons Reconocimiento-NoComercial-SinObraDerivada 4.0 Internacional 


\section{SECCIÓN RESEÑAS}

\section{Reseña. El desarrollo local en ámbitos rurales: experiencias y buenas prácticas para la empleabilidad y el emprendimiento}

Resumen: A continuación realizamos una breve crónica de lo que se expuso en la Mesa Redonda Virtual del pasado viernes 4 de diciembre de 2020, en la que se habló del Desarrollo Local en el medio rural y en la que participaron numerosas y numerosos ponentes expertos en la materia. Si hay algo que merece la pena destacar de lo aprendido en esta sesión, es que este ámbito profesional es sin duda, en primer lugar, una salida laboral con muchas oportunidades y, en segundo lugar, pero no por ello menos importante, una salida laboral que proporciona a sus profesionales una experiencia que va más allá de la estricta experiencia laboral, una experiencia vital y personal de la que todas y todos los ponentes hablaron ilusionados y encantados. Una salida laboral y un ámbito profesional -lo local y lo rural- que nos han explicado y hemos conocido poco a lo largo de estos cuatro años de carrera, pero que tan solo habiéndose tratado en este último año de nuestra formación de grado, se convierte en una de las más emocionantes.

Palabras clave: espacios rurales, desarrollo local, gobernanza, participación, aptitud.

Recibido: 08 de febrero de 2021

Devuelto para revisión: -

Aceptado: 09 de febrero de 2021

Referencia / Citation:

Quiles, P. J., Miguel, P., y Gil, G. (2021). Reseña. El desarrollo local en ámbitos rurales: experiencias y buenas prácticas para la empleabilidad y el emprendimiento. TERRA. Revista de Desarrollo Local, (8), 715-722. DOI 10.7203/terra.8.20357 


\section{Instituto Interuniversitario de Desarrollo Local, Universidad de Valencia \\ MESA REDONDA: EL DESARROLLO LOCAL EN ÁMBITOS RURALES. EXPERIENCIAS Y BUENAS PRÁCTICAS PARA LA EMPLEABILIDAD Y EL EMPRENDIMIENTO}

Valencia (España). Viernes, 04 de diciembre de 2020. Webminar desarrollado de 16h00 a $21 \mathrm{~h} 00$.

El hermoso consuelo de encontrar el mundo en un alma, de abrazar a mi especie en una criatura amiga

(Höderlin, 1998, p.43).

La mesa redonda fue inaugurada por su organizador y nuestro profesor de Desarrollo Local, el Dr. Jaime Escribano, quien nos introdujo en el contenido de la misma y nos expuso a grandes rasgos, que el Desarrollo Local es una gran oportunidad para los estudiantes de Ciencias Sociales en genera, y Ciencias Políticas en particular, tanto a nivel profesional como personal.

Así, a modo de presentación, fue la profesora Dra. María Dolores Pitarch, directora del Instituto Interuniversitario de Desarrollo Local de Valencia (IIDL-VLC), quien dio comienzo al acto explicando cómo las oportunidades -laborales o no- en el ámbito rural son la base para su desarrollo. Muchas veces, explica la profesora, el ámbito rural es un ámbito a considerar para mejorar la cohesión territorial, la igualdad de oportunidades y la equidad entre la dicotomía perversa que muchas veces se genera, de forma inapreciable y es un proceso connatural al hombre, entre el ámbito rural y el de las ciudades. Con ello, la Profa. Pitarch evidenció cómo la crisis sanitaria está teniendo un impacto negativo sobre los territorios rurales, lo que es fruto de desigualdades. De esto, ya conseguimos sonsacar la primera idea donde "el mundo en el que vivimos está reflejando que las nuevas tendencias globalizadoras tejen una serie de redes que cada vez se alejan más de lo local'".

Un pequeño apunte de ello viene de la mano del libro "España capital París", donde Germà Bel nos evidencia cómo en España no se ha llevado a cabo un desarrollo territorial integrado, sino que se resaltan los contrastes de un modelo radial y policéntrico, donde cada vez hay una mayor conexión e interoperabilidad entre las grandes ciudades, que han aglutinado la mayoría de los servicios, pero a su vez se ha dejado de lado lo que algunos nombran como la "Laponia Española". La Profa. Pitarch señala que el trabajo del IIDLVLC se especializa, precisamente, en cómo resolver tales desigualdades con investigaciones interdisciplinares y la colaboración entre actores de entidades públicas y privadas para mejorar la calidad de vida en entornos locales/rurales. Explica pues, que no buscan imponer su criterio, sino crear sinergias entre la academia y los territorios, dando a conocer buenas prácticas como es el caso de la mesa redonda que se estaba inaugurando en ese preciso instante.

Adentrándonos en las conferencias, en primer lugar, debemos hacer referencia a la intervención de Dña. Eulàlia Ribes, Agente de Empleo y Desarrollo Local (AEDL) de Banyeres de Mariola (Alicante) (Figura 1). En ella, Eulàlia expuso su visión personal sobre la figura del AEDL a partir de experiencia laboral en los distintos municipios en los que ha estado empleada, haciendo especial énfasis en el modo de trabajo que más y mejor 
le ha funcionado, la participación proactiva y directa sobre/con la población local, y el acceso a establecer sus costumbres, hábitos y rutinas como una manera más de conocer el territorio. Ella señaló la trascendencia de realizar esta labor de conocimiento de las estructuras sociales de la sociedad y ciudadanía, como punto de partida a la hora de poner en marcha proyectos sostenibles y resolutivos para el municipio. También nos insistió en la importancia de la participación ciudadana en las labores del ayuntamiento y en la función de puente que realiza el AEDL entre la ciudadanía y la corporación municipal, a la hora de implementar las políticas públicas pertinentes. Por último, nos destacó el papel de interacción que el AEDL mantiene con los cargos públicos, especialmente en los municipios pequeños, ya que esta labor participativa y cognitiva se hace todavía más importante para el correcto desarrollo de estos territorios.

Figura 1. Organización y ponentes de la mesa redonda

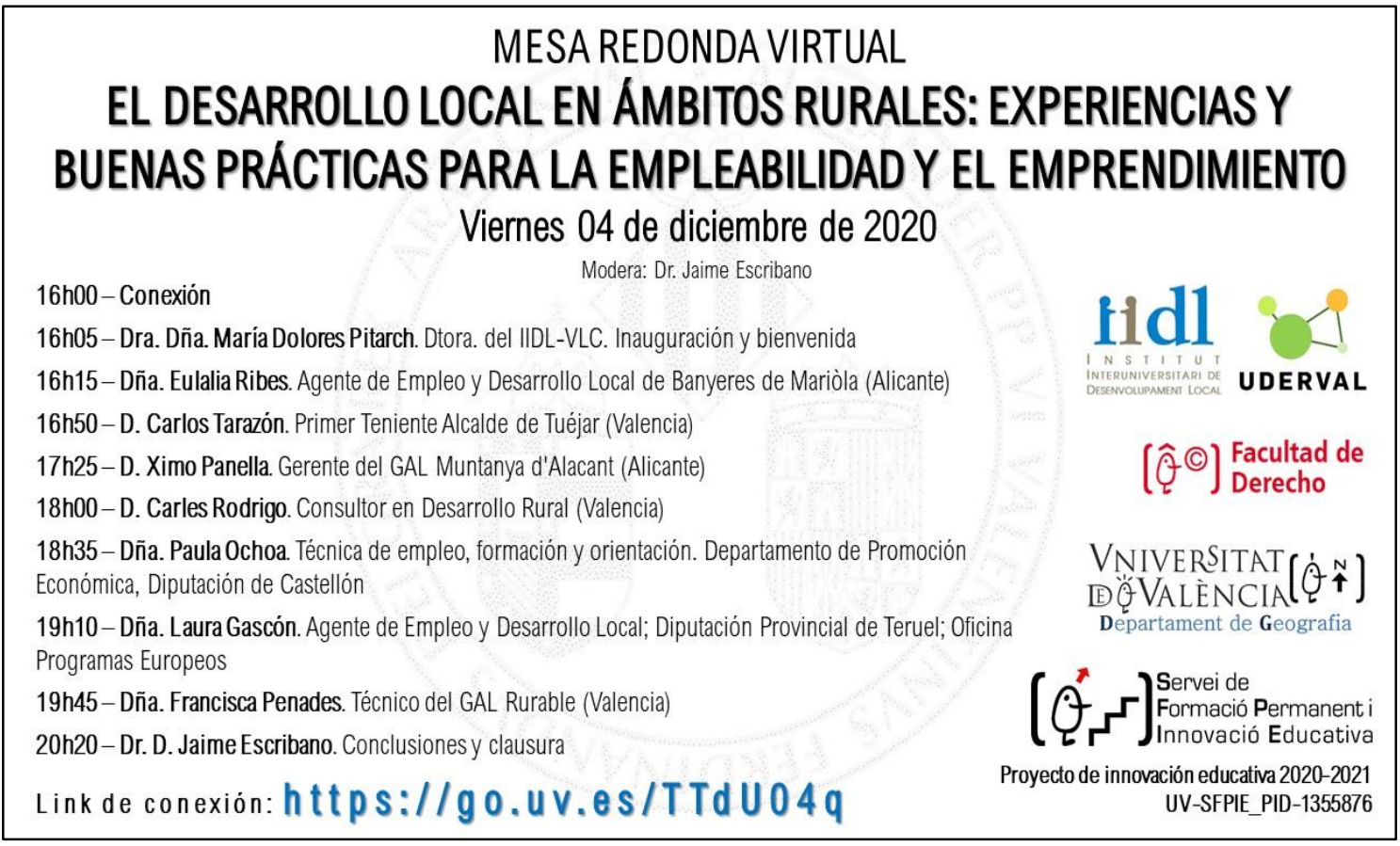

Fuente: Jaime Escribano.

Tras Eulàlia, intervino en segundo lugar D. Carlos Tarazón, primer Teniente de Alcalde del municipio serrano de Tuéjar (Valencia). Carlos comenzó hablando de "lo político", ensalzando el papel de la política para mejorar las vidas de la gente, en este caso en el mundo rural. En ese sentido, nos contó su experiencia en la corporación municipal de Tuéjar, que hasta hace poco tiempo estaba liderada por el Partido Popular y, por lo que nos comentó, los ciudadanos estaban relativamente descontentos con la gestión desarrollada en los últimos años. En consecuencia, se conformó un grupo de actores locales con diferentes ideologías en pro del municipio, con enfoques de trabajo y perspectivas de desarrollo renovadas, que pusieron de manifiesto cómo la ideología influye de una forma muy distinta en estos espacios, en relación a otros ámbitos como los urbanos, y, al margen de las propias discrepancias ideológicas de la agrupación que se conformó, esta se presentó a las elecciones municipales bajo unas siglas en común y fueron elegidos por el conjunto de la ciudadanía. A partir de entonces, Carlos, junto al resto del equipo de gobierno actual, ha tratado de conjugar el denominado enfoque del 
Desarrollo Local con el propio o relacionado con el Desarrollo Rural, puesto que para él tiene este territorio, el rural, tiene unas características diferentes de cualquier otro, abarcando en la actualidad, por ejemplo, muchos más aspectos que lo estrictamente agrario. En este sentido, os trasladó su idea sobre el medio rural y su desarrollo, en tanto en canto para él, tiene que ver con ensalzar el territorio, con "acabar con el paradigma de que lo rural como sinónimo de retraso". Para ello, nos insistió en, por ejemplo, que se ha de poner el foco en lo forestal, una cuestión que para él queda abandonada y que según afirma es un "nicho de mercado muy importante", al igual que la ganadería y la agricultura ecológica. En este sentido, Carlos apuntó las oportunidades que existen también en el ámbito de los servicios a la población, poniendo de ejemplo a Gestalgar y la cooperativa de mujeres de reciente creación, centrada en el cuidado de personas dependientes ${ }^{1}$. También añadió que el teletrabajo, que se está fomentando debido a la pandemia, es una oportunidad para estos municipios, para lo que sin duda es necesario potenciar y mejorar la conectividad previamente. Por último, se refirió a la formación profesional, pues desde su posición en el equipo de gobierno durante estos pocos años que llevan gestionando el municipio, ha detectado una falta importante de profesiones en empleos habituales, como por ejemplo fontaneros, cerrajeros, ebanistas, etc. Por último, finalizó su intervención con una frase que nos pareció especialmente reveladora de su filosofía y modo de entender los problemas rurales: "Geografía para entender el mundo, Política para cambiarlo".

En tercer lugar, intervino D. Ximo Panella, gerente del Grupo de Acción Local (GAL) "Muntanya d'Alacant" (Alicante). Ximo nos mostró los diferentes proyectos que se han ido llevando a cabo con ayuda de los fondos LEADER, herramienta fundamental para la diversificación productiva y la animación de la población del medio rural. Destacamos la importancia dada durante la exposición sobre la labor de dinamización social que realizan los GAL en este tipo de territorios. Una dinamización basada en el carácter transversal de los asistentes y participantes en los proyectos, como forma de integrar al mayor número de actores representativos en el mundo rural. Hizo énfasis en la importancia del relevo generacional, es decir, la atracción de los jóvenes en los proyectos y su implicación en las cuestiones de su entorno. También se señaló la necesidad de la cooperación en el ámbito rural, especialmente desde la economía, creando sinergias en sectores productivos como el turístico y el agrícola que generan un gran abanico de beneficios aparte del económico, como un capital social con raíces en el territorio.

En cuarto lugar, participó D. Carles Rodrigo, único representante del sector privado de toda la mesa redonda, lo que sin duda fue un plus, ya que nos aportó un punto de vista poco común en este tipo de encuentros: la del emprendedor "privado" en el ámbito rural. Nos contó su dilatada experiencia vital en la generación y gestión de proyectos de desarrollo territorial, y cómo ha transcurrido su vida laboral entre la Universidad, el ayuntamiento de Valencia (en sus inicios) y diferentes ayudas públicas para acabar creando su propia empresa de consultoría privada. En este sentido, nos enumeró las principales actividades que viene desarrollando en los últimos años, desde promover procesos participativos, hacer memorias de viabilidad, charlas de experiencia, etc. Un sinfín de labores donde si tuviéramos que extraer alguna idea, más allá de la energía que nos transmitió -también requisito indispensable para emprender en el territorio rural-, fue la de multidisciplinariedad. Un consultor privado en el ámbito rural debe ser capaz de todo y no debe dejar pasar ninguna oportunidad de negocio. Por ello, nos reiteró

\footnotetext{
${ }^{1}$ Se puede conocer más de esta iniciativa a partir de la información recogida en la siguiente noticia de prensa: https://www.levante-emv.com/videos/comunitat-valenciana/2018/06/11/cooperativa-ayudadomicilio-dependientes-gestalgar-11271696.html (recuperada el 05 de febrero de 2021).
} 
acertadamente que "si tus conocimientos te limitan, debes tirar mano de colaboradores", enfatizando con ello la importancia de crear y participar en redes.

La siguiente ponente, quinta en intervenir en la mesa redonda, fue Dña. Paula Ochoa, Técnica del Departamento de Promoción Económica de la Diputación de Castellón. Fue estudiante de Ciencias Políticas y Sociología y, posteriormente, curso el antiguo Máster en Gestión y Promoción del Desarrollo Local que ofertaban conjuntamente la Universitat de València y la UJI de Castellón. Desde su posición actual, nos contó su labor de dinamización económica de todo el territorio de la provincia de Castellón. Así, incidió en la divergencia existente entre las zonas costeras y las zonas de interior de la provincia, formado estas últimas por una gran mayoría de municipios rurales que son el objetivo primordial del departamento en el que ella realiza sus labores. Concretamente, nos explicó el funcionamiento de los Centros CEDES de dinamización, como son los ubicados en Albocàsser, Morella, Segorbe, Vall d'Alba y Llucena, enfocados todos ellos en la dinamización económica de las comarcas en los que se encentran, a través de distintas actividades que nos comentó en su exposición. En estas zonas, se realizan planes bianuales que recogen actuaciones, seleccionadas de forma participativa y colectiva con el municipio, que consideran que son necesarias para dicha dinamización, tanto para personas desempleadas (formación con el objetivo de capacitar a las personas de dichos territorios), como para personas emprendedoras (con servicios de asesoramiento a las mismas), como para empresas ya existentes (sobre todo, de cara a su dinamización y modernización). Durante su intervención, nos proporcionó numerosos ejemplos de proyectos realizados en estos municipios. Si algo creemos que debemos de extraer de las palabras de Paula, es que "existen posibilidades y herramientas para promover el crecimiento económico y la calidad de vida de la población rural, que no es una misión perdida o fallida, que son municipios que cada vez se articulan más como una alternativa a la alta concentración de población en las grandes ciudades de la costa castellonense". Tras ello, nos contó su experiencia personal acerca de cómo ha llegado al puesto en el que está, algo sin duda muy enriquecedor para aquellos -como nosotros- que estamos cursando una formación parecida.

En sexto y sétimo lugar intervinieron, en el siguiente orden, Dña. Laura Gascón, AEDL de la Diputación Provincial de Teruel en la Oficina de Proyectos Europeos, y Dña. Francisca Penades, Técnico del GAL "Rurable" (Valencia). Ambas nos contaron su experiencia profesional, incidiendo especialmente en algo que ya había sido apuntado también con anterioridad, y que nos resultó especialmente llamativo por desconocerlo completamente: la precariedad -temporal- de estos perfiles profesionales. Por ejemplo, Laura nos enumeró los diversos empleos por los que ha pasado en los últimos años hasta llegar al puesto que ocupa en la actualidad, subrayando la concatenación de contratos anuales. Y Francisca indicó que en su aventura como AEDL, aventura que dura más de 40 años, se estancia media en los territorios en los que ha trabajado se ajusta a una duración de tres años por empleo, empleo además muy dependiente de las subvenciones públicas, lo que en cierto modo, incrementa la vulnerabilidad del AEDL.

Esto nos lleva a reflexionar sobre hasta qué punto, esta precariedad y dependencia condiciona las labores diarias de un AEDL o un técnico de GAL, ya que bajo nuestra formación en el ámbito de los partidos, sindicados, lobbies y demás viguerías del sistema político, uno de sus objetivos -sino el primordial- es el de su perpetuación en el tiempo, "caiga quien caiga" si se nos permite la expresión. Para nuestra sorpresa, Francisca nos respondió que "la condición de temporalidad es algo que ya se tiene asumido cuando se empieza esta carrera profesional, y que en todo caso, en vez de incentivar la 
individualidad para que se mantengan estas figuras por mérito propio es un incentivo hacia el buen hacer de su profesión" (Figura 2).

Figura 2. Intervención de Francisca Penades

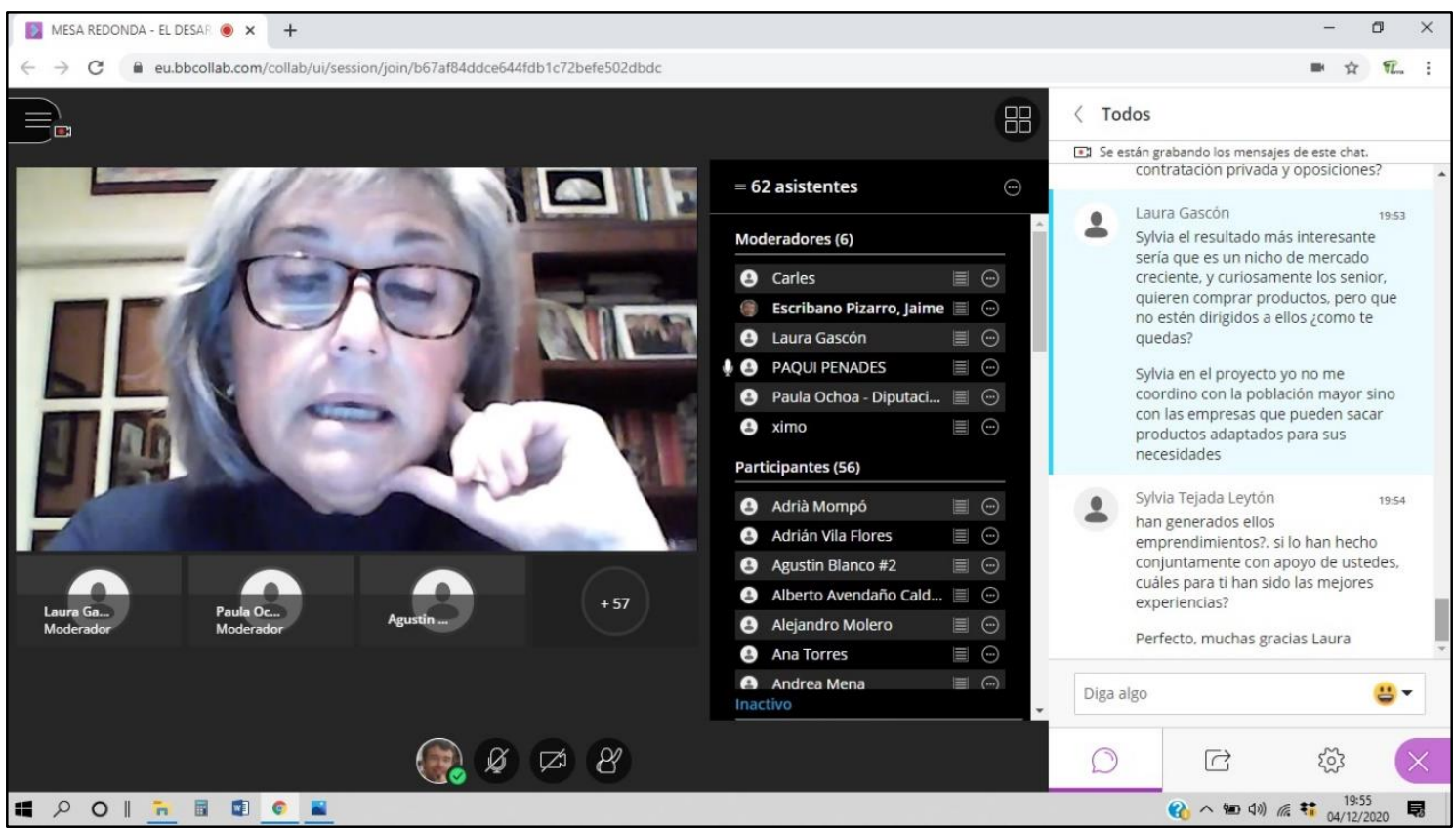

Fuente: Jaime Escribano.

Una vez finalizadas las intervenciones de los siete ponentes invitaos, señalamos como uno de los aspectos más relevantes desde nuestro punto de vista, las múltiples menciones que se hicieron sobre las relaciones intercooperativas, y la necesidad de crear redes, sinergias y lógicas inclusivas. En particular, a partir de planteamiento en donde se consiga la participación efectiva y comprometida de todo tipo de actores, e intentando integrar su diversos intereses de modo que los resultados sean beneficiosos para un proyecto de desarrollo común, guiado y liderado conjuntamente por el sector público y el privado. Y, además, con un enfoque supralocal, capaz de dejar atrás la habitual "política de campanario". Tal vez, esta forma de ejercer el desarrollo local centrada en las interrelaciones sea la que mejor responde a un enfoque de crecimiento neoendógeno, es decir, un modelo de desarrollo que tenga en cuenta los recursos propios de lo local, pero que a su vez necesita de actores extralocales que incorporen activos que puedan generar un impacto positivo en el territorio.

Por último, no podemos dejar de lado la aportación al "saber-hacer" que pueden realizar a la materia y/o disciplina del Desarrollo Local, específicamente, los graduados en Ciencias Políticas y de la Administración Pública. Los "politólogos" digamos que somos una suerte de "navaja suiza", tenemos conocimiento de varias ciencias, tanto como la Sociología, el Medio Ambiente, la Economía, la Geografía, el Derecho, del sistema político que nos envuelve y de la administración en su conjunto. Nuestra formación nos aporta una visión multidisciplinar del campo sobre el que se sitúa el conflicto, y la originalidad para resolver problemas y/o saber identificarlos se sitúa como fortaleza en nuestra formación. De hecho, como señala Farinós (2016, p.45), "la planificación territorial no es sólo una disciplina académica o una cuestión ideológica, sino que 
también es una profesión con tradición, dedicada al noble arte de procurar alternativas y propuestas acerca de cómo procurar el mejor desarrollo de las comunidades".

En conclusión, tras unas últimas palabras de nuestro profesor Jaime Escribano para cerrar el encuentro, hemos podido ser partícipes de una iniciativa académico-formativa muy productiva y esperanzadora para estudiantes como nosotros, que además de estar interesados por la Ciencia Política como se puede desprender de nuestros estudios, también versamos un importante interés sobre el Desarrollo Local y el municipalismo, sobre la importancia del nuevo paradigma de lo local en el futuro, donde la globalización y la eliminación de cada vez más fronteras ponen en el centro a cada uno de los territorios locales. En este encuentro hemos podido observar las distintas oportunidades que se nos abre a cada uno de nosotros y nosotras en este sentido, viendo en cada una de las experiencias tratadas distintas formas de dedicar una vida a este trabajo, todas ellas con una persona entregada detrás que, pese a todas las dificultades encontradas en el camino, mantiene viva la ilusión y el esfuerzo por hacer de sus territorios un lugar mejor y mejorar así la vida de las personas que lo componen. Esto, para politólogos como los autores de esta reseña, es un descubrimiento que abre muchas puertas tanto profesionales como personales.

\section{REFERENCIAS}

Casellas, A. (2011). La crisis, la geografía económica y Julie Graham: alternativas al desarrollo local a partir de la crítica feminista. Anales de Geografía de la Universidad Complutense, 31 31-46. https://doi.org/10.5209/rev_AGUC.2011.v31.n2.37017

Farinós, J. (2016). Planificación territorial y desarrollo local, y su relación con las nuevas formas de gobernanza territorial asociadas. Un renovado espacio de aplicación profesional. En J. Noguera (coord.), La visión territorial y sostenible del desarrollo local: una perspectiva multidisciplinar (pp. 67-92). Publicaciones de la Universitat de València.

Bel, G. (2010). España, capital París. Destino.

Pere Joan Quiles Lledó, Pablo Miguel Argudo y Guillem Gil Puig

Graduados en Ciencias Políticas y de la Administración Pública, promoción 2017-2021.

Universitat de Valènca

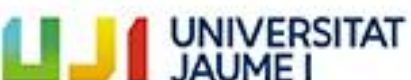

Institut Interuniversitari de Desenvolupament Local
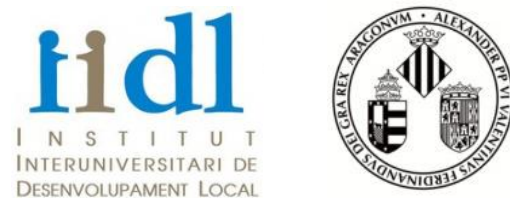

VNIVERSITAT (E) VAlÈnCia 